\title{
ARNAUD-DOMINIQUE HOUTE
}

\section{LE GENDARME NATIONAL \\ FACE À LA SOCIÉTÉ FRANÇAISE (1870-1914): DU SERVICE DE L'ÉTAT AU SERVICE DU PUBLIC?}

À la fin de l'année $1870^{\circ}$, dans l'Hérault, la nouvelle municipalité de Bédarieux fait abattre le monument aux morts dressé en 1852 à la mémoire des gendarmes tués par les insurgés de décembre $1851^{1}$. Cet événement est emblématique de la défiance, sinon de la haine, qu'éprouvent les républicains envers une institution inféodée à l'Empire déchu. Un peu plus de quarante ans plus tard, c'est pourtant cette même gendarmerie qui mène une mobilisation générale dont tous les observateurs soulignent la surprenante efficacité. Il y a loin du gendarme de 1850 à celui de 1900 . Le regard de la société s'est déplacé: là où la petite Mina d'Alexandre Dumas sanglotait à la simple pensée des gendarmes, Toinou les observe d'un æil rassuré ${ }^{2}$. Là où les moqueries de Gustave Nadaud restaient insidieusement subversives, les vaudevilles de la Belle Époque raillent l'uniforme sans intention maligne. Les coups de bâton de Guignol ont cessé d'effrayer le pouvoir ${ }^{3}$.

Pendant ce demi-siècle, la société a profondément changé. On pourrait gloser sur les nouvelles angoisses sécuritaires ou insister sur la libéralisation du système judiciaire ${ }^{4}$ : sans doute la population a-t-elle moins de raisons de se méfier de gendarmes plus étroitement bridés et plus directement utiles. Mais s'en tenir à ces explications reviendrait à méconnaître l'importante mutation engagée par la gendarmerie. Grâce à Jean-François Chanet, on connaît bien l'exemple des instituteurs, dont on sait qu'ils ont appris à conjuguer l'appren-

\footnotetext{
' Messager du Midi, 22 juin 1872.

${ }^{2}$ Alexandre DumAS, Les Mohicans de Paris, Paris 2001; Antoine SYlvère, Toinou, le cri d'un enfant auvergnat, Paris 1993, p. 277. Les représentations de la gendarmerie ne sont jamais univoques, mais on discerne assez nettement une tendance générale.

${ }^{3}$ Aurélien LignereuX, De la conquête du territoire à celle des cours? Présence et perception du gendarme dans la France du XIX siècle, DEA, histoire, dir. Jean-Noël Luc, université Paris IV, 2002, p. 41-100. Au début du XX $\mathrm{XX}^{\mathrm{e}}$ siècle, syndicalistes et socialistes condamnent fréquemment l'action répressive de la gendarmerie, mais l'institution n'est pas vraiment contestée. Peut-être tire-t-elle un profit indirect de son rôle actif lors des inventaires des biens du clergé.

${ }^{4}$ Dominique Kalifa, L'encre et le sang. Récits de crimes et société à la Belle Époque, Paris 1995; Jean-Claude FARCY, L'histoire de la justice française de la Révolution à nos jours, Paris 2001.
} 
tissage de la nation et l'entretien des particularismes locaux, la société englobante et la communauté ancienne ${ }^{5}$. Or, plus encore que les autres institutions d'État, la gendarmerie doit gagner la confiance des citoyens et conquérir la légitimité démocratique qui lui fait cruellement défaut. Pour y parvenir, l'institution doit changer de comportement: de nouveaux regards sur le métier et sur la société, de nouvelles manières d'agir et de penser, voilà ce qui transforme les prétoriens de l'Empire en gendarmes de la République.

Reste à savoir comment l'on modifie l'identité professionnelle d'une institution forte de plusieurs siècles d'histoire et d'une vingtaine de milliers de membres. Le poids des héritages s'ajoute, en effet, à la variété et à la dispersion des hommes pour brouiller le tableau. À plus forte raison, la gendarmerie du XIX ${ }^{\mathfrak{e}}$ siècle ne dispose pas d'un commandement unifié spécifique qui soit susceptible de lancer une impulsion directrice ${ }^{6}$. Sous de nombreux aspects, il vaudrait d'ailleurs mieux parler de gendarmeries, en fonction des régions et des niveaux hiérarchiques. Les directives réglementaires ne suffisent donc certainement pas à expliquer la transformation du métier. Pour comprendre cette mue démocratique, il faut délaisser les discours officiels et "passer d'une conception substantialiste de la signification aux usages « ${ }^{7}$. Représentant l'État, les gendarmes en véhiculent une image spécifique, en partie indépendante des conceptions politiques et juridiques et de leurs traductions normatives. C'est donc sur cette communauté d'individus qu'il faut recentrer l'objectif afin d'observer "au ras du sol ${ }^{8}$ l'une des modalités de la construction d'un État démocratique moderne?

${ }^{5}$ Jean-François CHANET, L'École républicaine et les petites patries, Paris 1996.

${ }^{6}$ Depuis la suppression de l'Inspection générale de la gendarmerie, en 1815 , jusqu'à la création d'une Direction de la gendarmerie, en 1918, l'arme est placée sous le contrôle des généraux de la cavalerie, qui ne s'en soucient guère. Dans cette situation, les stratégies institutionnelles cèdent le pas aux tactiques individuelles.

${ }^{7}$ Philippe CORCUFF et Claudette LAFAYE, Éclairages locaux sur les transformations des services publics. À la croisée des niveaux micro- et macro-sociaux, Commissariat Général du Plan, février 1992, p. 50.

${ }^{8}$ Cette célèbre formule de Jacques Revel rappelle les mérites, sinon d'une micro-histoire difficile à pratiquer sans sources adaptées, du moins d'une histoire localisée.

${ }^{9}$ Cette communication s'inscrit dans le cadre de la préparation d'une thèse de doctorat, Le métier de gendarme national au $\mathrm{XIX}^{\mathfrak{e}}$ siècle: la construction d'une identité professionnelle (1851-1914), dir. Jean-Noël Luc, université Paris IV. 
Les bras armés de l'État

\section{Le drapeau au village}

Depuis la loi fondatrice du 28 germinal an VI, la gendarmerie est »instituée pour veiller à la sûreté publique et pour assurer le maintien de l'ordre et l'exécution des lois; une surveillance continue et répressive constitue l'essence de son service ${ }^{10}$. Dotée d'attributions policières, elle n'en appartient pas moins pleinement à l'armée. C'est cette ambiguïté d'un régime mixte, farouchement défendu tout au long du siècle, qui distingue l'arme des autres forces de l'ordre. À l'opposé des gardes champêtres ou des agents de police, les gendarmes ne dépendent pas des autorités locales. Ils ne sont même pas assujettis, du moins dans les textes, aux institutions civiles: lorsqu'un magistrat ou un préfet désire s'assurer le concours de la gendarmerie, il doit recourir à la formule contraignante de la réquisition. La seule allégeance reconnue par toutes les compagnies départementales reste donc le service de l'État. C'est ainsi que le corps échappe, au moins partiellement, aux tiraillements centrifuges des intérêts locaux.

Seule force de police véritablement nationale et relativement indépendante, la gendarmerie dispose également de l'implantation la plus homogène. Dispersés en petites unités de quatre à six hommes, les gendarmes sont répartis sur l'intégralité du territoire. À compter de 1850 , le principe d'une brigade par canton est adopté. Il est très rapidement appliqué et ne souffre guère d'exceptions à la chute de l'Empire. Présente dans chaque chef-lieu de canton, fréquemment encasernée dans un lieu central et bien matérialisé, souvent voisine de la mairie ou de la justice de paix, la gendarmerie fait donc partie de ces institutions de voisinage que connaissent tous les habitants. Le fonctionnement du service renforce d'ailleurs cette logique de proximité. En effet, la tournée quotidienne constitue l'essentiel du travail des gendarmes. Selon un calendrier rigoureux, ceux-ci doivent parcourir les routes et les villages jusqu'aux moindres hameaux. Chaque commune doit être visitée au moins deux fois par mois. En raison de cette contrainte, la surveillance préventive l'emporte le plus souvent sur l'action répressive. Ni les impératifs policiers, ni les velléités personnelles, ne peuvent éloigner trop longtemps le gendarme du quadrillage de sa circonscription.

Aux yeux des villageois, la brigade constitue donc bien l'une des premières incarnations de l'État, l'un de ses visages les plus familiers. Elle en livre une

${ }^{10}$ Cette expression scande les principaux textes réglementaires, notamment le décret du $1^{\text {er }}$ mars 1854 et le décret de 1903. Pour toutes les références officielles, on renvoie une fois pour toutes au Mémorial de la gendarmerie, recueil annuel dont la publication remonte au milieu du $\mathrm{XIX}^{\mathfrak{e}}$ siècle. 
image protéiforme, beaucoup plus complexe qu'il ne semble. Bien sûr, la mission policière l'emporte, et le procès-verbal reste l'unité de mesure par excellence de l'activité professionnelle. Engagés en première ligne dans le combat de l'acculturation nationale et juridique des communautés, les gendarmes prennent alors la figure d'un État dur et répressif. Mais, dès le Second Empire, d'autres facettes du métier se développent. La défense des biens et des personnes prend une importance renforcée dans une société dont les seuils de tolérance $s^{\prime}$ abaissent ${ }^{11}$. Le renseignement juridique et le conseil militaire restent une nécessité dans un contexte d'alphabétisation restreinte et de judiciarisation croissante des conflits. Enfin, la protection contre les calamités naturelles devient une attente de plus en plus prégnante ${ }^{12}$. À l'image de l'État qu'ils représentent, les gendarmes doivent maintenir un équilibre entre prévention et répression. Cependant, à l'orée de la Troisième République, le balancier penche assez nettement.

\section{Une lecture guerrière du métier?}

La gendarmerie du Second Empire s'est largement construite sur l'événement fondateur du coup d'État ${ }^{13}$. En effet, face aux résistances qui se sont éveillées en décembre 1851, les gendarmes ont pris place parmi les premiers défenseurs du prince-président. Victimes des insurgés pour certains, artisans de la répression pour la plupart, ils ont tiré profit de cet événement fondateur pour acquérir une reconnaissance politique et sociale qui les fuyait depuis la chute du Premier Empire. Lorsque l'on sait que la majorité des gendarmes en activité en 1870 ont été recrutés au début des années 1850 , on comprend sans peine la coloration bonapartiste de l'institution. Si tous ne sont pas de fervents "zélotes « du régime impérial, beaucoup n'en partagent pas moins une lecture prétorienne du métier, fondée sur le souvenir du coup d'État, comme si la légitimité de la gendarmerie n'apparaissait qu'au miroir des guerres civiles. Cette conception nourrit encore fortement l'imaginaire professionnel au début des années 1870. La répression de la Commune de Paris, en partie menée par des gendarmes, contribue à l'entretenir et même à la réactiver. Il faut tenir compte de cette perception quasi-guerrière du métier pour comprendre le fonctionnement quotidien des brigades.

"Alain CORBIN, L'histoire de la violence dans les campagnes françaises au XIX siècle: esquisse d'un bilan, dans: Ethnologie française, 1991-1993, p. 224-236.

${ }^{12}$ Cyril CARTAYRADE, Assister et secourir au XIX ${ }^{\mathrm{e}}$ siècle: l'exemple des gendarmes du Puyde-Dôme, dans: Revue de la Gendarmerie Nationale, hors-série 2 (2000) p. 49-53.

${ }^{13}$ Pour des informations complémentaires, cf. Arnaud-Dominique HouTE, La gendarmerie au miroir de Décembre: Cochet de Savigny et la légitimation du coup d'État, dans: Sylvie APRILE et al. (dir.), Comment meurt une République. Autour du 2 décembre 1851, Paris 2004, p. 201-210. 
En particulier, cette mythologie professionnelle peut éclairer le rapport des gendarmes à l'impopularité de leur travail policier. Bien sûr, aucun procèsverbal n'est jamais bien accueilli. Mais certains le sont peut-être moins que d'autres. Or, la gendarmerie semble acquérir, sous le Second Empire, le monopole des interventions les plus difficiles. Le canton de Solre-le-Château en offre un exemple éloquent. Trois autorités policières y coexistent: le commissaire cantonal et ses agents, une dizaine de gardes champêtres et cinq gendarmes. $\mathrm{Au}$ vu des archives, leur collaboration fonctionne sans heurt majeur, et un partage des tâches s'opère plus ou moins naturellement. Pourtant, l'équilibre apparent cache des spécialisations significatives. Ainsi, la brigade est particulièrement active dans la police du roulage. Au contraire du commissaire cantonal, qui ne relève pratiquement que des infractions de stationnement, notifiées après coup, les gendarmes s'exposent directement à la colère des voituriers verbalisés. Plus présents également dans les affaires de tapage et de violence, ils jouent surtout un rôle ingrat dans la surveillance des cabarets. Si les agents de police sanctionnent surtout les instruments de mesure défaillants et l'ivresse publique, les gendarmes sont les seuls à s'attaquer aux débits de boisson ouverts après l'heure légale de fermeture ${ }^{14}$. Plus que toute autre, cette mission obscure alimente des rancœurs durables et mobilise les communautés locales contre un corps qui ignore les aménagements coutumiers. On retrouve ici la spécificité fondamentale de la gendarmerie: peu soucieuse du respect des arrêtés municipaux, dont elle laisse l'application aux gardes champêtres, l'arme prend une part active à l'acculturation des campagnes. Sans doute cette posture ingrate s'alimente-t-elle aux racines identitaires du métier. Mais, pour imprégner vraiment les esprits sous les bicornes, il faut encore qu'elle s'accorde au positionnement social des individus.

Or, les gendarmes ne sont pas vraiment des horsains. Sociologiquement, ils ne diffèrent guère de la société qui les accueille ${ }^{15}$. Sauf rares exceptions, ils sont issus des classes les plus modestes de la société. Massivement ruraux, ils sont majoritairement affectés dans leur région de naissance ${ }^{16}$. D'ailleurs, l'extrême fréquence des mariages sur place renforce l'intégration à la population surveillée. Dans ces conditions, on s'explique mal le succès d'une conception répressive, sinon guerrière, du métier. Certes, les plus convaincus sont d'abord les nouveaux promus: moins liés aux populations et soucieux de se

\footnotetext{
${ }^{14}$ Tribunal de simple police de Solre; Archives départementales [désormais A.D.] du Nord, 4 U $14-69$ et 73.

is Tous les renseignements prosopographiques proviennent des registres matricules de contrôles des troupes; Service Historique de l'Armée de Terre (SHAT) Vincennes, sous-série $42 \mathrm{Yc}$. On se reportera aussi à Louis SAUREL (capitaine), La gendarmerie dans la société de la Seconde République et du Second Empire, thèse, Sorbonne, 1957, 7 vol.

${ }^{16}$ Il faut toutefois prendre garde de ne pas confondre le recrutement régional - qui suppose un certain brassage - et le recrutement local, très rare dans la gendarmerie, au contraire d'autres administrations.
} 
faire remarquer, ils multiplient les contrôles et les tracasseries. Mais l'enthousiasme des premiers temps ne s'estompe pas forcément, et certains persistent à heurter de front les habitudes installées. L'arrogance de ces hommes est trop communément dénoncée pour qu'on ne cherche pas à l'expliquer. On peut alors avancer l'hypothèse selon laquelle la posture orgueilleuse de ces gendarmes traduit un désir de notabilité. „C'est moi seul qui suis le maître de Solesmes«, clame le brigadier Guenne en traversant le bourg au grand galop ${ }^{17}$. D'autres exemples témoignent d'une même volonté de s'afficher comme des coqs de village. Fondée sur une position sociale avantageuse et sur une certaine fierté de l'uniforme, cette morgue déborde le champ clos du métier pour envahir l'arène publique. En affrontant le maire et les notables, les gendarmes se grandissent. Au nom du service de l'État, ils essayent de se placer au-dessus de la société. Mais cette entreprise se paye d'un isolement trop douloureux pour que les uns ne préfêrent pas y renoncer, tandis que la chute de LouisNapoléon fragilise les autres.

\section{De la délégitimation politique à la crise d'identité}

Associée dans l'opinion publique au régime déchu, la gendarmerie paye le prix des faveurs passées. Dès l'été 1870 , on signale les premières manifestations hostiles à l'arme. Le 4 septembre, la foule menace les gendarmes qui défendent l'Assemblée. Crémieux s'emporte: „On veut donc provoquer le peuple que l'on se sert de la gendarmerie! Cet uniforme lui est odieux ${ }^{18}$. La revanche républicaine reste toutefois modérée par les impératifs de sécurité publique. Dès 1871 , en particulier avec l'avènement de Thiers et après la Commune, la gendarmerie retrouve, sinon son lustre, du moins son utilité. Lorsqu'il s'agit de réorganiser complètement l'armée, rares sont ceux qui contestent l'existence de l'institution ${ }^{19}$. Aussi se contente-t-on de maintenir le corps en l'état. En dépit de cet apaisement, la gendarmerie souffre d'une réelle crise d'identité. Le choc de 1870 vient, en effet, raviver le souvenir des révolutions de 1830 et de 1848 , durant lesquelles l'arme s'était déjà retrouvée du mauvais côté des barricades. Pire encore, l'identification bonapartiste de l'institution alourdit le poids de la défaite. De plus en plus nombreux, officiers et observateurs s'efforcent de repenser une gendarmerie dont les fondements ont volé en éclats.

\footnotetext{
${ }^{17}$ Lettre anonyme au préfet du Nord, juin 1858; A.D. Nord, 5R 27.

${ }^{18}$ Cité par Pierre MiQuel, Les Gendarmes, Paris 1990, p. 252.

${ }^{19}$ Xavier BORDA, Gendarmerie et statut militaire: les projets de démilitarisation de la gendarmerie sous la Troisième République, dans: Jean-Noël LUC (dir.), Gendarmerie, État et société au XIX' siècle, Paris 2002, p. 91-99.
} 
De ces débats, les gendarmes ne perçoivent qu'un écho assourdi. La presse professionnelle et les discours officiels masquent l'ampleur de la crise, d'autant que l'insistance mise sur la Commune semble renouveler le mythe identitaire du Deux-Décembre. Mais, sur le terrain, la situation s'assombrit. Les conflits locaux se généralisent, et ils prennent une ampleur inconnue auparavant. En cette période de flou constitutionnel, tout se passe comme si chaque lieu de pouvoir cherchait à marquer un avantage sur ses rivaux. Placés dans une position particulièrement exposée, les gendarmes sont d'autant plus vulnérables que le nouveau régime les couvre moins franchement. Et l'entrée en scène des républicains renverse l'équilibre des forces. La fin des années 1870 voit ainsi une explosion des affrontements locaux entre maires et brigades. La dénonciation politique sert alors souvent de prétexte à des règlements de comptes entre instances concurrentes. En 1880, une enquête nationale est même diligentée auprès des préfets, chargés de dresser un bilan des relations entre les municipalités et la gendarmerie ${ }^{20}$. Invitées à aplanir les dernières hostilités, les autorités départementales utilisent à fond l'arme des mutations. $\mathrm{Au}$ final, l'épuration reste très limitée, mais les gendarmes ont clairement perdu la bataille de la notabilité. Le jeu s'est retourné contre eux.

Désormais, c'est donc la culture de l'accommodement qui doit l'emporter. I1 faut dire que ce mode de fonctionnement n'est pas complètement étranger aux gendarmes. Les rapprochements sociologiques et familiaux entraînent une bonne partie du corps à manier plus prudemment le langage du sabre et à privilégier la prévention ou, plus exactement, la répression ciblée. Une fois de plus, l'exemple de Solre-le-Château le confirme: peut-on encore parler de hasard lorsque la majorité des procès-verbaux dressés pour contravention à la police du roulage visent des étrangers à la circonscription? De toute évidence, les gendarmes savent fermer les yeux lorsque leur intégration est en jeu ${ }^{21}$. La proximité exerce un travail de sape sur le long terme. Au cours des années 1870 , elle rencontre la crise des modèles hérités pour transformer profondément l'institution. Bras affaibli de l'État, la gendarmerie ne peut plus - ne veut plus - être l'adversaire de la société.

${ }^{20}$ Plusieurs dépôts d'archives départementales en gardent la trace; notamment A.D. Gironde, 5R 18.

${ }^{21}$ Les sociologues ont développé une analyse comparable pour la période contemporaine; Renée ZAUBERMAN, La répression des infractions routières: le gendarme comme juge, dans: Sociologie du travail 1 (1998) p. 43-64. 


\section{À nouveaux gendarmes, nouvelles pratiques: \\ les chemins de la démocratisation}

\section{Les gendarmes de la République}

Cette mutation s'impose d'autant plus facilement qu'elle coïncide avec un profond renouvellement du corps. En effet, la génération recrutée autour de 1851 termine sa carrière au début des années 1870 . Ce hasard démographique se double d'une politique volontariste d'augmentation des effectifs: 425 brigades sont créées entre 1872 et 1878 . Les nouveaux recrutements atteignent donc un niveau suffisant pour transformer la physionomie du corps. Même si l'encadrement se compose encore de sous-officiers et d'officiers plus expérimentés, généralement formés sous l'Empire, les germes d'un renouveau apparaissent. D'ailleurs, les pics d'indiscipline constatés à la fin de la décennie témoignent sans doute d'une lutte des générations. Politiques et professionnels, ces affrontements marquent un temps de transition.

Rajeunie, la gendarmerie des années 1870 ne diffère pourtant guère de son ancêtre. Le recrutement continue à privilégier le monde rural et les classes les plus modestes. Le brassage géographique reste limité dans le temps et dans l'espace. On continue à éviter les affectations trop locales, mais la part des vrais horsains semble se restreindre significativement. Il faut dire que l'institution peine à accueillir des candidats de valeur. L'évolution des critères de recrutement en témoigne: la taille minimale est abaissée, de même que l'expérience militaire requise; le niveau d'instruction exigé reste stable, alors même que l'alphabétisation de la population s'est développée. Même en tenant compte de l'écart entre les normes et les usages, il est sans doute plus facile de devenir gendarme sous la Troisième République. Dans ces conditions, l'homologie sociale de la gendarmerie et de la population se renforce d'autant plus que les motivations des recrues changent également.

En la matière, les sources sont rares ${ }^{22}$, mais on peut quand même distinguer plusieurs raisons pour endosser l'uniforme. Première d'entre elles, la vocation ne jouait et ne joue qu'un rôle marginal. Au contraire, le désir d'achever tranquillement sa carrière militaire a sans doute décidé de nombreux vieux militaires à rejoindre la gendarmerie. Mais, à partir des années 1870 , les avantages réservés aux soldats rengagés facilitent leur maintien au régiment. D'ailleurs, la durée moyenne des services militaires des gendarmes diminue constamment. Cette filière de recrutement s'affaiblit donc fortement, au profit d'une autre logique de carrière: la possibilité d'attendre un autre emploi. De plus en

\footnotetext{
${ }^{22}$ Les rares autobiographies de gendarmes sont recensées dans: Jean-Noël LUC (dir.), Guide de recherches en histoire de la maréchaussée et de la gendarmerie, Service Historique de la Gendarmerie Nationale, 2005.
} 
plus, surtout à la fin du siècle, les gendarmes ne gardent l'uniforme qu'une dizaine d'années, ce qui suffit à leur donner droit à une modeste retraite proportionnelle et leur ouvre l'accès à des emplois réservés. La gendarmerie devient progressivement une profession d'attente, un sas de sortie du monde militaire et de retour dans la vie civile. "Ma première intention était d'atteindre dans cette arme quinze ans de services, puis de postuler un emploi réservé«, reconnaît Julien Duhamel, recruté peu avant $1914^{23}$. Peu importe que la plupart décident finalement de garder l'uniforme: la mutation des motivations initiales engendre naturellement une modification des comportements. Du vieux soldat de la première moitié du siècle à son héritier, le futur civil, c'est tout un monde de représentations qui change. De plus en plus, le profil du gendarme se banalise.

Du reste, l'entrée dans la gendarmerie s'explique d'abord et surtout par des avantages matériels. Solde régulière, sécurité de l'emploi, possibilités d'avancement, droit à la retraite... Toutes ces caractéristiques du métier peuvent séduire des candidats issus des couches populaires. Pourtant, les gendarmes se plaignent fréquemment de manquer du nécessaire. Apparue très tôt dans le siècle, la complainte ne cesse pas avec la Belle Époque. Au contraire, les revendications se développent et dénoncent tout particulièrement la constante dégradation du niveau de vie. Évidemment, une forte part d'exagération, difficilement quantifiable, nourrit ces récriminations intéressées. Elles répondent, sinon à une réalité, du moins à un sentiment très vif. Si le gendarme du Second Empire pouvait encore faire figure de petit notable, son successeur ne fait plus illusion. La solde progresse beaucoup moins vite que les autres salaires. Au milieu du XIX ${ }^{\mathrm{e}}$ siècle, la position sociale du gendarme l'assimilait aux commissaires de police ou aux agents supérieurs des douanes. Au début du $\mathrm{XX}^{\mathrm{e}}$ siècle, sa fortune se compare à celle des facteurs ou des gardes forestiers. Fût-elle relative, cette dépréciation matérielle engendre une dévalorisation symbolique dont témoignent les difficultés de recrutement de la Belle Époque. Les nouveaux gendarmes sont d'autant plus proches de la société qu'ils sont moins susceptibles de s'en distinguer.

\section{Dépolitisation et professionnalisation}

Après l'effondrement des fondements identitaires bonapartistes de la gendarmerie, l'arme est obligëe de redéfinir son socle de valeurs. Dans un premier temps, l'avènement de l'ordre moral repousse le temps des décisions. Contrôle des opposants, surveillance des élections, renseignements sur l'opinion publique, toutes ces missions quotidiennes des brigades perdurent; seuls les interlo-

23 Julien DUhamel (capitaine), Mémoires d'un officier de gendarmerie (8 avril 18863 janvier 1975), ms, fol. 2 . 
cuteurs changent. Cependant, les rouages du système policier se grippent. Nostalgiques de Louis-Napoléon, certains gendarmes répugnent plus ou moins ouvertement à servir le nouveau régime. D'autres dénoncent le principe même de ces interventions politiques. Ce faisant, ils s'inscrivent d'ailleurs dans une longue tradition cristallisée dans les textes réglementaires: sans cesse réitérée depuis 1820 , l'interdiction des déguisements ne vise pas seulement à distinguer l'arme des Vidocq policiers, elle limite surtout l'utilisation des gendarmes pour l'espionnage. Avec l'avènement d'un régime auquel l'arme est moins attachée, on comprend que ces réticences s'expriment plus clairement. Dès l'automne 1870, le colonel de Mondésert refuse d'enquêter sur les sympathies politiques des fonctionnaires corses, car »la gendarmerie ne peut, ni ne doit faire de police occulte $\ll^{24}$. Dès lors, toutes les réflexions sur la gendarmerie moderne insistent sur son désengagement nécessaire.

Il faut toutefois attendre la chute de l'ordre moral. Au même titre que tous les autres fonctionnaires, les gendarmes sont invités à défendre les députés conservateurs. En Ille-et-Vilaine, une circulaire du chef de légion explique que »la cause du libéralisme outré [prône] la suppression de l'armée et de toute force répressive«. Dans leur propre intérêt, les gendarmes sont donc tenus d'appuyer les candidats de Mac-Mahon. Transmise à Gambetta et lue à la tribune de la Chambre des députés ${ }^{25}$, cette note scandalise l'hémicycle et conforte l'opinion publique dans ses préjugés. Après la victoire des républicains, les interrogations redoublent de vigueur. Cette fois, la gendarmerie compte bien se désengager définitivement des implications politiques qui l'ont durablement affaiblie. Le discours réglementaire porte la marque de cette résolution. Dès 1878, le ministre de la Guerre recommande aux gendarmes de "s'abstenir de tout acte ou de toute parole ayant trait à la politique«. Trois ans plus tard, il précise que "la gendarmerie doit se tenir soigneusement en dehors de toute lutte électorale ${ }^{26}$. Évidemment, les actes contredisent parfois ces beaux principes. Mais la vigilance des journaux professionnels, attentifs à signaler toute dérive, témoigne d'un basculement des mentalités. La gendarmerie se dépolitise vraiment.

L'ancien serviteur du pouvoir cherche alors de nouvelles allégeances. Puisque l'État ne se confond plus avec le gouvernement en place, il lui faut une nouvelle incarnation que la gendarmerie va trouver dans la sacralisation des règlements. Ainsi passe-t-on du légitimisme politique au légalisme réglementaire. La formule est bien connue d'une institution habituée aux coups d'État et aux révolutions. Dès 1848 , Cochet de Savigny en faisait une maxime du corps: „que l'on soit en monarchie ou en république, la gendarmerie est tou-

\footnotetext{
${ }^{24}$ Note sans date; SHAT, 5 Yf 75924.

${ }^{25}$ Discours prononcé à la Chambre des députés, le 15 novembre 1877, par Léon Gambetta, Paris 1877, p. 25-26.

${ }^{26}$ Circulaires du ministre de la Guerre aux chefs de légion, 4 avril 1878 et 20 juillet 1881 .
} 
jours l'arrière-garde de la loi « ${ }^{27}$. Cette vieille tradition du repli professionnel n'en trouve pas moins une nouvelle jeunesse. En témoigne la teneur des historiques rédigés durant les années 1880 , qui tentent tous de restituer la continuité d'une institution que les vicissitudes politiques se contentent d'effleurer ${ }^{28}$. La disparition du serment politique et le maintien d'un serment professionnel confortent également cette mutation. D'ailleurs, les représentations dominantes peignent désormais l'image d'un gendarme obsédé par ses règlements.

Plus profondément, la dépolitisation s'inscrit dans le mouvement de long terme de la professionnalisation du corps. En l'occurrence, il ne s'agit pas seulement d'améliorer la formation ou le recrutement, ni même de renforcer l'esprit de corps. Il faut surtout consolider une autonomie encore fragile en libérant la gendarmerie de la tutelle des institutions politiques et militaires. Mais les autorités craignent avant tout d'ouvrir la boîte de Pandore de la démilitarisation. C'est pourquoi, contexte aidant, l'accent se porte sur la dépolitisation totale du corps. Dès les années 1880 , la seconde professionnalisation de la gendarmerie se manifeste donc par une re-militarisation du corps censée la prémunir des dérives civiles. Les inspecteurs généraux vantent alors ces gendarmes »jaloux de s'affirmer comme étant d'abord des soldats ne s'immisçant en aucune façon dans des questions touchant la politique « ${ }^{29}$. Développement des exercices proprement militaires, renforcement de la discipline et stricte tutelle des autorités hiérarchiques éloignent sans doute le spectre bonapartiste. Ces mesures ne satisfont pas pour autant les gendarmes qui veulent désormais acquérir la maîtrise de leur métier. Très populaire, le mot d'ordre de »la gendarmerie aux gendarmes « s'installe dans les discours ${ }^{30}$. Plus discrètes, des traductions pratiques s'en font jour. Ainsi de ces gendarmes sétois, insultés par un pauvre diable, qui choisissent de le verbaliser sans l'incarcérer: ce laxisme provoque la colère du commandant. Mais les gendarmes se défendent »en raison de la latitude que [leur] laisse le >Dictionnaire Cochet de Savigny، et aussi en raison que [le prévenu] conduisait une voiture de déménagement chargée de mobilier à destination de Pézenas et afin d'éviter tout retard « ${ }^{31}$. L'épisode

\footnotetext{
27 Journal de la Gendarmerie, $\mathrm{n}^{\circ} 106,1^{\text {er }}$ avril 1848.

${ }^{28}$ En plus des historiques généraux publiés par plusieurs officiers supérieurs, les compagnies sont désormais invitées à rédiger et à tenir à jour un historique régimentaire. Dans tous ces ouvrages, la chronologie politique s'efface sous la tradition professionnelle.

29 Ordre laissé à la $7^{e}$ légion par le général inspecteur Germer-Durand, repris par L'Écho de la Gendarmerie, 19 août 1900.

${ }^{30}$ Il est tout particulièrement popularisé à la Belle Époque par les retraités de la gendarmerie. On se permettra de renvoyer à notre article: Arnaud-Dominique HOUTE, Un métier en crise? La gendarmerie de la Belle Époque d'après ses retraités, dans: Sociétés et Représentations 16 (2003), Figures de gendarmes, p. 153-166.

${ }^{31}$ Rapport du chef de brigade de Cette au commandant d'arrondissement, 27 décembre 1904, SHGN, 34 E 529. Le Dictionnaire de la gendarmerie initié par Cochet de Savigny rassemble les principaux textes réglementaires de l'arme.
} 
est particulièrement significatif: appuyés sur une bible réglementaire plurivoque, les gendarmes se considèrent les meilleurs juges de leur travail. Le développement de l'implantation de proximité vient d'ailleurs renforcer cette lecture corporatiste de la professionnalisation.

\section{L'ouverture au public}

Si les missions officielles de la gendarmerie ne changent guère, le métier s'adapte progressivement au mouvement de démocratisation. Désormais, le peuple souverain dispose d'un droit de regard, au moins théorique, sur ses forces de police. À leur modeste niveau, les brigades de gendarmerie n'échappent pas à ce nouvel ordre du monde. Il faut dire que l'obligation de prendre en compte la demande publique ne bouleverse pas vraiment leurs pratiques. D'une part, les gendarmes n'ont jamais pu complètement négliger les attentes de leurs administrés. Refuser de les entendre aurait d'ailleurs condamné leur travail de police judiciaire et de collecte du renseignement. D'autre part, la vox populi reste bien timide et se cantonne, pour l'essentiel, aux revendications de quelques notables bien informés. De plus, le repli professionnel et militaire de l'institution facilite la surdité des échelons locaux: rien de plus facile que de renvoyer la requête aux supérieurs hiérarchiques. La presse régionale ne s'y trompe pas, qui préfère souvent s'en prendre aux policiers municipaux, plus facilement contrôlables. Dans ces conditions, la révolution démocratique de la gendarmerie se réduit pratiquement à un changement d'attitude.

Limitées, les évolutions n'en sont pas moins multiples et significatives. Plus encore qu'auparavant, la prudence commande les comportements professionnels: il s'agit avant tout d'éviter des affrontements et des scandales qui ne tourneraient pas forcément au profit de l'institution. C'est pourquoi, d'ailleurs, les gendarmes sont invités à policer leurs manières. Cette recommandation théorique n'est pas neuve, loin s'en faut ${ }^{32}$, mais elle devient plus contraignante et elle s'accompagne d'un réel effort de formation. Le développement des conférences morales permet de rappeler aux gendarmes les règles essentielles de la politesse. On constate également la rédaction de premiers manuels de savoir-vivre spécialement destinés aux officiers issus du rang. Quant aux gendarmes trop brusques, ils sont sanctionnés. Ainsi le chef d'escadron Brissaud prend en grippe la wbrigade des petits messieurs [de Luzarches], qui semble avoir une très haute idée de sa situation et de sa valeur «: »les écarts de langage, les propos maladroits et les tracasseries immotivées« provoquent un

\footnotetext{
${ }^{32}$ Les manuels rédigés sous les monarchies constitutionnelles accordaient une place majeure à ces questions.
} 
rappel à l'ordre des plus sévères ${ }^{33}$. Les brigades apprennent également à mettre en valeur leurs résultats. Comme dans d'autres compagnies, en dépit des consignes officielles, les registres de procès-verbaux des Hautes-Alpes sont ainsi communiqués aux journalistes afin d'orienter les articles de presse ${ }^{34}$. Dans d'autres cas, une priorité quasi-publicitaire guide l'organisation du service. Il faut montrer l'uniforme pour effrayer le délinquant, mais aussi pour rassurer le bon citoyen. Au-delà de cette image répressive, la gendarmerie entre également plus volontiers sur la scène communautaire. Lors des fêtes publiques ou des manifestations, les gendarmes ne se contentent plus de maintenir l'ordre. Ils participent pleinement aux festivités. En Sologne, Maurice Genevoix enfant décrit longuement l'impressionnante parade militaire de la brigade de gendarmerie du lieu ${ }^{35}$. Les meetings sportifs ou les courses automobiles exigent également leurs gendarmes de prestige. Comme la publicité, l'apparat fait partie du métier de gendarme en démocratie.

Toutes ces mutations. s'imposent lentement. Elles prennent appui sur la transformation progressive du lien entre les gendarmes et leurs administrés. Si la caserne de la première moitié du siècle pouvait encore être assimilée à une citadelle, sinon à une tour $d^{\prime}$ ivoire ${ }^{36}$, son héritière gagne en familiarité ce qu'elle perd en solennité. Dès le Second Empire, par endroits, les habitants prennent l'habitude de venir directement déposer plainte. Le plus souvent, toutefois, on continue à attendre le passage des gendarmes ou à informer le maire ou son garde champêtre. Parfois trop distante, souvent trop intimidante, la caserne reste retranchée du bourg. Mais le mouvement de rapprochement est bien engagé, et les réformes militaires de la Troisième République lui donnent certainement un nouvel élan. En effet, à compter des années 1870, quand le principe de la conscription s'impose, le statut militaire de la gendarmerie la qualifie tout naturellement pour contribuer à la gestion quotidienne des réservistes et des territoriaux. La brigade devient la première interlocutrice du citoyen en armes. Obligés de transmettre nommément les ordres d'appel et d'instruire les demandes de dispense ou d'ajournement, les gendarmes y trouvent certes un surcroît de travail, mais ils sont astreints à mieux connaître les lieux et les personnes. Quant à leur caserne, elle doit gagner en capacité d'accueil. Reste à savoir comment les gendarmes accueillent ces innovations.

${ }^{33}$ Rapports au chef de légion, 8 février et 26 mai 1899; SHAT, dossier Brissaud, 10 Yd 1512.

${ }^{34}$ Rapport du chef d'escadron, 17 février 1899; SHGN, 5E 2. De manière générale, les officiers sont très attentifs à toutes les critiques provenant des journaux.

${ }^{35}$ Maurice Genevoix, Au cadran de mon clocher, Paris 1969, p. 72.

${ }^{36}$ Frédéric Chauvaud, Les passions villageoises au $\mathrm{XIX}^{\mathfrak{e}}$ siècle: les émotions populaires dans les pays de Beauce, du Hurepoix et du Mantois, Paris 1995, p. 120. 
Des pratiques aux valeurs:

l'identité professionnelle des gendarmes de la Belle Époque

\section{La crise de la Belle Époque: une mise à l'épreuve}

En empruntant le virage de la démocratisation, la gendarmerie se heurte à des difficultés imprévues que la dernière décennie du XIX ${ }^{\mathfrak{e}}$ siècle met en lumière. Détournés de leurs fonctions répressives traditionnelles au profit d'attributions moins strictement policières, les gendarmes sont accusés de laisser le champ libre aux malfaiteurs de toutes sortes. Les crimes de Vacher, cet assassin vagabond qui traverse la France sans être inquiété, choquent profondément l'opinion et provoquent un vaste débat sur la sûreté publique. L'utilité de la gendarmerie est remise en question, on lui reproche sa placidité et son archaïsme ${ }^{37}$. Il faut dire que le bicorne et le baudrier du gendarme à cheval font piètre figure face aux exploits médiatiques des "Brigades du Tigre«. Voilà donc la rançon de la familiarisation: le gendarme n'intimide plus. Suffisamment conscients de ces problèmes pour ne pas imputer la crise sécuritaire au seul laxisme des magistrats, les gendarmes s'inquiètent surtout du retard technique accumulé et des détournements de fonction. Avec le développement du maintien de l'ordre aux grèves, il n'est pas rare, au début du $\mathrm{XX}^{\mathrm{e}}$ siècle que les brigades soient réduites à deux ou trois hommes ${ }^{38}$. Encore faut-il qu'elles mènent des enquêtes administratives toujours plus nombreuses... Le constat de crise est dressé précisément et relayé grâce au porte-voix des comités de retraités. Mais tous ne s'entendent pas sur les remèdes à apporter.

En simplifiant, trois grandes voies s'ouvrent alors à la gendarmerie ${ }^{39}$. La première s'inspire des projets de création d'une gendarmerie mobile. Il s'agirait de replier l'arme sur son statut militaire et sur les missions de maintien de l'ordre. Mais ce choix irait à l'encontre des principes de territorialisation et de familiarité. C'est pourquoi une majorité de gendarmes mènent l'opposition aux projets de mobile. Parmi les plus ardents militants, certains prônent même une réorientation inverse de la gendarmerie. Il s'agirait de moderniser franchement l'institution et d'y réduire l'emprise militaire pour la transformer en police judiciaire d'élite. Là aussi, même si un modèle policier

\footnotetext{
${ }^{37}$ Jean-Marc BERLIÈrE, La gendarmerie en question au début du XX ${ }^{e}$ siècle, dans: Jean-Noël LuC (dir.), Gendarmerie, p. 101-116.

${ }^{38}$ Lors du demier trimestre 1903 , toutes les brigades de la $1^{\text {ere }}$ légion sont réduites à un ou deux hommes: »le fonctionnement de la police rurale a été à peu près entièrement suspendu«; lettre du ministre de l'Intérieur au ministre de la Guerre, 12 mai 1905, SHAT, 7N 44.

${ }^{39}$ Cette idée est développée dans notre communication au colloque La Gendarmerie au XX' $X^{e}$ siècle (université Paris IV, juin 2003): Arnaud-Dominique HouTE et Aurélien LIGNEREUX, Du bicome au képi: les gendarmes de la Belle Époque à la croisée des chemins, actes à paraître en 2006.
} 
remplace le modèle casqué, l'argument de proximité est battu en brèche. Dans les deux cas, on a bien affaire à des polices de souveraineté, pour lesquelles prime le service de l'État. Or, la plupart des gendarmes ne sont pas prêts à cette révolution conceptuelle. Ils défendent donc, sinon un statu quo intenable, du moins le maintien de l'équilibre traditionnel: force nationale, mais localisée, la gendarmerie doit se fonder sur la proximité, la polyvalence et le service conjugué de l'État et du public. Cette définition reprend les principes de la loi du 28 germinal an VI. Mais elle en renouvelle l'argumentation. Ainsi, le port de l'uniforme ne représente plus tant l'allégeance à une tutelle étatique lointaine qu'une marque de transparence coutumière et rassurante. L'infléchissement est significatif de la victoire d'une nouvelle conception du métier, empirique, forgée dans les décennies de la République triomphante.

\section{Au service du public: statut professionnel et implantation sociale}

Comment expliquer la rapidité de cette acculturation? Un dernier détour s'impose pour comprendre comment les gendarmes jouent au quotidien des concepts généraux. En effet, sans s'y réduire, la culture professionnelle est aussi le masque de l'intérêt privé. C'est ce que comprennent d'ailleurs bien les généraux chargés d'étudier la faisabilité des régiments mobiles. Selon ces observateurs, les fortes réticences du corps s'expliquent d'abord par la peur de perdre un ancrage social: »ce qu'il faut [aux gendarmes], c'est la brigade, le mariage, un logement et un jardin ${ }^{40}$. De fait, ces avantages acquis comptent suffisamment pour que certains gendarmes renoncent à obtenir une promotion qui les obligerait à changer de poste. Et la mutation dans l'intérêt du service figure parmi les punitions les plus redoutées. La sédentarisation des individus constitue donc bien l'un des principaux et des plus anciens attraits du métier. Mais ce goût de la stabilité rencontre l'hostilité des officiers, qui craignent non sans raisons - les compromissions et dénoncent l'apathie des gendarmes trop bien installés dans leur canton. Les commandants qui engagent la lutte restent toutefois isolés. L'application de la circulaire qui interdit aux gendarmes d'exercer dans le canton de leurs parents laisse à désirer. Lorsqu'un gendarme se marie dans sa résidence, on demande sa mutation seulement quand la profession de la belle-famille peut créer des difficultés justifiées. Par conséquent, les brigades se composent de plus en plus souvent d'individus réellement liés au canton et à leurs administrés.

Cette adéquation de la nouvelle doctrine aux vœux des gendarmes rencontre également les besoins du service. On a vu combien le souci du public se développait. Dès lors que les gendarmes appartiennent vraiment à la société qu'ils

${ }^{41}$ Note préparatoire à la séance du Conseil supérieur de la Guerre, 24 avril 1913. SHAT, IN 11 . 
surveillent, cette préoccupation légitime leurs liens extra-professionnels. Dans le pire des cas, lorsque le gendarme oublie la dignité de son uniforme, la bonhomie excuse l'incompétence: comme dans le conte d'Octave Mirbeau, wle prestige de la gendarmerie se trouvait bien un peu diminué par toutes les frasques de Barjeot, mais il était si bon enfant, si peu gendarme, ce sacré lascar de Barjeot, qu'on n'y faisait point attention ${ }^{41}$. La littérature est féconde de ces figures de gendarmes joviaux, qui n'arrêtent guère de malfaiteurs, mais qui jouent un rôle social tout aussi éminent: c'est en grande partie à ces mythes que l'institution doit la popularité qu'elle recherche de plus en plus ${ }^{42}$. D'ailleurs, l'institution ne se prive pas de les développer elle-même. Voici l'exemple que le lieutenant Carrez propose à ses hommes, "ce vieux gendarme de la vieille école, $[\ldots]$ ayant accompli la majeure partie de sa carrière dans la même résidence, au point que plusieurs générations avaient grandi sous son règne $[\ldots]$ : ce vieux gendarme était un oracle, car sa sagesse et sa pondération s'étaient affirmées bien souvent ${ }^{43}$. Dans cette nouvelle conception du métier, la manière d'être compte autant, sinon plus, que la manière de faire: l'exemplarité commande la vie quotidienne des gendarmes. La gendarmerie se découvre un rôle social ${ }^{44}$, et le gendarme s'y forge une raison sociale.

Il ne suffit pas, en effet, de s'intégrer aux populations; encore faut-il y trouver une place honorable. Or, on l'a vu, le niveau de vie des gendarmes perd en valeur relative, tandis que leur uniforme se banalise: ni l'argument financier, ni le prestige militaire, ne suffisent désormais à distinguer le gendarme de ses concitoyens. Par contre, l'exercice du métier garantit une stature et offre une position de surplomb sur la société. Qu'il s'agisse d'interpeller des vagabonds menaçants ou d'arrêter un cheval emballé, la gendarmerie peut prouver son utilité publique. Les colonnes des journaux en attestent, les brigades jouent un rôle discret, mais efficace, dans ces petites actions de préservation de l'ordre établi. Le gendarme reste également un agent assermenté, susceptible de verbaliser; mais il est surtout celui qui peut fermer les yeux ou trouver un arrangement. Il délivre un avis motivé sur l'opportunité d'accepter telle ou telle demande de sursis militaire; mais il se contente généralement d'apostiller la

${ }^{41}$ Octave MIRBEAU, Le gendarme, L'Écho de Paris, $1^{\text {er }}$ octobre 1889 , réédité dans: Contes cruels, Paris 1990, p. 362-363.

${ }^{42}$ Selon Georges Maire, wc'est une banalité de constater qu'ils sont plus sympathiques et plus populaires que les gardiens de la paix «, Georges MAIRE, Étude sur la gendarmerie par Georges Maire et un gendarme en activité de service, Paris 1905, p. 1.

${ }^{43} \mathrm{~J}$. CARREZ (lieutenant), Un mercredi par mois. Causeries morales ou pratiques à l'usage des militaires de la gendarmerie, Paris 1912, p. 144.

${ }^{44}$ Arnaud-Dominique HOUTE, Les officiers, les gendarmes, la nation. Autour du rôle social de l'officier dans la France de la Belle Époque, dans: Revue de la gendarmerie nationale 206 (2003) p. 111-115. 
requête d'un avis favorable ${ }^{45}$. Le pouvoir policier et administratif est d'autant mieux accepté et d'autant plus respecté qu'il s'exerce au bénéfice de la population $^{46}$. C'est en appliquant spontanément ce précepte que les gendarmes conquièrent une position sociale avantageuse. En exerçant le métier à leur manière, ils devancent et accompagnent la démocratisation de l'institution.

D'une identité professionnelle à l'autre, on retrouve donc la trame générale de la démocratisation. À l'orée de la Troisième République, les gendarmes entretiennent les derniers feux d'une conception guerrière du métier. Deux générations plus tard, ils défendent une pratique de proximité que la crise sécuritaire met à mal. Ces quelques décennies n'ont pourtant pas bouleversé le quotidien des brigades. Au contraire, que l'on observe la sociologie du corps, la réalité de ses missions ou le fonctionnement de son service, les traits communs l'emportent largement. Ces similitudes alimentent d'ailleurs le reproche d'archaïsme qui s'abat sur la gendarmerie de la Belle Époque. Cependant, le regard sur le métier a changé. Aussi bien au milieu du XIX ${ }^{\mathrm{e}}$ siècle qu'au début du $\mathrm{XX}^{\mathrm{e}}$ siècle, le gendarme assure le service de l'État et du public. Ce dédoublement d'allégeance engendre des contradictions qu'il résout en fonction des contextes d'action et de son intérêt personnel, mais aussi en fonction de sa socialisation professionnelle. Avec l'avènement républicain et les mutations rapides des années 1870 et 1880 , les nouvelles valeurs démocratiques rencontrent le repositionnement social des gendarmes sur le terrain d'entente de la proximité. Pour eux, le balancier penche désormais vers le "service du public«, au moment même où s'invente le concept républicain de "service public ${ }^{47}$ : principes généraux et pratiques quotidiennes s'entrecroisent. Et c'est pourquoi il faut suivre l'injonction de Michel Foucault de "saisir le pouvoir du côté de l'extrémité de moins en moins juridique de son exercice « ${ }^{48}$ pour percevoir cette républicanisation progressive de l'administration. Car les intérêts corporatistes et l'adaptation aux attentes publiques l'emportent sur l'action consciente des dirigeants. Pour autant, il faut bien reconnaître que ce choix de la proximité sert finalement la construction de l'État. Les gendarmes n'ont pas

${ }^{45}$ De mai 1906 à mai 1907, la brigade de Châteaudun ne renvoie qu'un avis défavorable aux demandes d'ajournement des réservistes convoqués pour leurs périodes d'exercice; SHGN, carton 780. Très peu de gendarmes prennent le risque d'encourir la rancune de la population. Ainsi, "s'il y a grève, la vie du brigadier Mac [qui a refusé d'apostiller une demande de dispense] sera en danger «; rapport du commissaire spécial, $1^{\text {er }}$ décembre 1904, A.D. PyrénéesOrientales, 12176.

${ }^{46}$ Bien évidemment, cette négociation de la sécurité publique ignore les catégories marginalisées qui, exclues du jeu, risquent d'en devenir les boucs émissaires; voir, par exemple, Jean-François WAGNIART, Le vagabond à la fin du XIX siècle, Paris 1999. On retrouve ici le débat sur les déficits démocratiques de la III ${ }^{\mathrm{e}}$ République.

${ }^{47}$ Vida AZIMI, Le service public, dans: Vincent DUCLERT, Christophe PROCHASSON (dir.), Dictionnaire critique de la République, Paris 2002, p. 252-253.

${ }^{48}$ Michel FouCAULT, »Il faut défendre la société«, Paris 1997, p. 25. 
rompu avec l'éthique militaire de l'obéissance qui reste, au contraire, un principe identitaire fort. Simplement, la traduction des directives s'est assouplie, l'espace de la transaction s'est élargi. Ce faisant, les gendarmes ont sans doute gagné en esprit de responsabilité et en influence ${ }^{49}$ plus qu'ils n'ont perdu en rigueur et en rectitude.

\section{Deutsche Zusammenfassung}

Die Figur des "gendarme " als traditionelle Verkörperung staatlicher Hoheit erfährt in der zweiten Hälfte des 19. Jahrhunderts bedeutende Veränderungen. Um 1870 ist die "gendarmerie « noch mit einer Prätorianergarde gleichzusetzen, eng verbunden mit dem kaiserlichen Regime und kaum in die Gemeinschaft integriert, die sie überwacht. 40 Jahre später bildet sie jedoch eine der Hauptstützen der Republik. Sie ist in allen Regionen verwurzelt und daran gewöhnt, im Namen des Nachbarschaftsprinzips eher mit Vertrauen als mit Furcht betrachtet zu werden. Es handelt sich hier um ein generelles Modell der Befriedung der Beziehungen zwischen Staat und Gesellschaft, verbunden mit der Verbreitung der Idee des öffentlichen Dienstes.

Während dieser Zeit hat sich in der beruflichen Praxis keine wirkliche Veränderung ergeben. Denn es sind weniger das soziale Profil, der militärische Status oder die Arbeit der "gendarmes«, sonderm es ist vielmehr die eigene Einschätzung ihrer Aufgaben, die sich wandelt. Entpolitisierung und wohlwollende Einstellung gegenüber der Öffentlichkeit sind die sichtbarsten Anzeichen eines generellen Wechsels, der sich besonders im Verhältnis zu den Mitbürgern offenbart: Der hochmütige und scharfe Ton tritt hinter Professionalität zurück, durchsetzt von einem gewissen $\mathrm{MaB}$ an Gutmütigkeit. Das Bestreben des "gendarmes« geht nicht mehr dahin, sich durchzusetzen, sondern akzeptiert zu werden.

Wie ist diese fundamentale Wandlung des beruflichen Selbstverständnisses zu erklären? Zweifelsohne rührt sie nicht her von einer Strategie, die die Obrigkeit entworfen hätte. Sie ist vielmehr das Ergebnis einer Taktik, die die "gendarmes« angesichts neuer politischer und sozialer Gegebenheiten selbst entwickeln. Die so verstandenen Interessen und verinnerlichten Werte begleiten nicht nur den republikanischen Umbruch bei der Wahrung der öffentlichen Ordnung, sondern gehen ihm sogar voraus.

49 Les mémoires du gendarme Vermeren mettent en lumière l'éthique de responsabilité d'un agent de l'ordre colonial; Henri VerMEREN, Un gendarme aux colonies, mémoires commentés par Patrice Vermeren, Paris, 2003, en particulier p. 253. 